\section{Pilot Testing of an Out-of-Country Medical Care Questionnaire with Screening and Cost Analysis of Preemptive Isolation for Carbapenem-Resistant Enterobacteriaceae in a Large Canadian Health Region}

To the Editor-The spread of carbapenem-resistant Enterobacteriaceae (CRE) has become an important global public health concern. Since their emergence, numerous institutional outbreaks have been reported worldwide..$^{1-3}$ Infections with these multidrug-resistant organisms have limited treatment options and are associated with significant morbidity and mortality. ${ }^{4,5}$ The lack of systematic surveillance for CRE in many countries presents a major prevalence knowledge gap. A key risk factor for CRE acquisition is the receipt of medical care in countries that are considered areas of endemicity for CRE. ${ }^{6}$ Prompt identification and isolation of patients infected or colonized with CRE has been advocated as a means for preventing transmission in hospital settings but has significant resource implications. ${ }^{1,2,5}$

Using a focused out-of-country medical care (OCMC) screening questionnaire, we determined the proportion of admitted patients in our health region (4 hospitals with a catchment area of approximately 1.4 million people ${ }^{7}$ ) who received OCMC in the previous 12 months, assessed their CRE colonization status, and estimated the costs associated with a preemptive isolation strategy. The pilot project took place over a 2-month period between July and September 2012 , a period chosen to capture both the peak tourist season and local residents returning from their summer vacations. We found $42 \%$ and $52 \%$ of overnight person-visits made by US and overseas visitors, respectively, occur between July and September each year within our region. ${ }^{8}$ However, transborder and international travel from Calgary residents have peaks in both the winter and summer months. The questionnaire inquired about location and type (inpatient vs outpatient) of OCMC. It was administered by the admitting clerk or nurse at the time of hospital admission and then sent to the Infection Prevention and Control (IPC) offices at each site. IPC practitioners used our electronic order entry system to order rectal swab samples or stool samples for patients identified as having received OCMC within the previous 12 months. Samples were tested for the presence of CRE using CHROMagar KPC media.

Costs for preemptive isolation were estimated from previously published data on isolation costs for extendedspectrum $\beta$-lactamase-producing gram-negative organisms in a large Canadian hospital. ${ }^{9}$ Costs were estimated for a 3day period of isolation (based on our usual reporting time) on the assumption that most patients would have negative test results for CRE, allowing isolation to be discontinued once test results were available. Cost analysis took into consideration the additional costs associated with a private room, contact precautions (additional nursing time and supplies), IPC time, and additional housekeeping time. The final figure was adjusted by a factor of 1.16 to reflect the Bank of Canada inflation rate between 2005 and 2013. ${ }^{10}$

During the 2-month screening period, there were 13,835 admissions. Screening questionnaires were administered to 6,646 patients (48\% of admissions). Among all patients screened by questionnaire, $206(3.1 \%)$ were found to have received OCMC in the previous 12 months. A majority of patients received outpatient medical care (59\%), which is likely associated with a lower risk of becoming colonized with CRE on the basis of the known risk factors of indwelling medical devices and cumulative healthcare or antibiotic exposure, which are likely to be more significant in an inpatient population. Inpatient hospitalizations made up 18\% (37 patients), whereas $16 \%$ (33 patients) received care in both inpatient and outpatient settings. The most common locations were the United States (34\%), Asia (23\%), Europe (15\%), and Central and South America (11\%). These results were not surprising given our proximity to the United States and the large Asian immigrant population in our region. Both of these settings are considered to be areas of CRE endemicity. CRE screening samples were obtained for 101 patients (49\% of those who received OCMC), and none were colonized with CRE. Extrapolation to a full year yields 2,573 OCMC recipients, which may be an overestimate, but if we project that the summer months represent approximately half of all outof-country and local resident out-of-country travel, the extrapolation yields 1,286 OCMC recipients. Using this figure, which may be an underestimate given the midwinter travel by local residents, the costs for preemptive isolation for 3 days ( $\$ 925$ per patient) would be $\$ 1.19$ million per year. The cost of isolating only recipients of inpatient OCMC (including those who were cared for in both inpatient and outpatient settings) would be $\$ 404,688$ per year.

This study was subject to several limitations. The reasons for the $48 \%$ completion rate for the screening questionnaires were multifactorial and included limited awareness of the study by some frontline staff responsible for questionnaire administration, the additional time commitment required to complete the form in a time- and resource-constrained work environment, and patient factors, such as language barriers or critical illness that precluded administration of the questionnaires. The most commonly cited reason for failing to collect a CRE screening sample from a patient was that the patient was discharged from the hospital before sample collection. We acknowledge the difficulty of using extrapolation to determine annual rates of OCMC but provided a conser- 
vative figure in our calculations. We also recognize that the general inflation factor used may be an underestimate for "medical" inflation.

With increasing rates of travel and medical tourism, more patients are receiving OCMC. Although this point-prevalence study did not identify any CRE-colonized patients, ongoing surveillance and stringent infection control practices will be critical for identifying and limiting the spread of CRE among hospitalized patients in Canada. A preemptive isolation strategy has significant resource implications and is not economically practical at this time in our setting given the low prevalence of CRE colonization.

\section{ACKNOWLEDGMENTS}

Potential conflicts of interest. All authors report no conflicts of interest relevant to this article. All authors submitted the ICMJE Form for Disclosure of Potential Conflicts of Interest, and the conflicts that the editors consider relevant to this article are disclosed here.

\section{Nipunie Rajapakse, $\mathrm{MD}^{1}$ Joseph Vayalumkal, $\mathrm{MD} ;{ }^{1,2}$ Debbie Lam-Li, BSc; ${ }^{2}$ Craig Pearce, MSc; ${ }^{2}$ Gwynne Rees, MSc; ${ }^{2}$ Linda Kamhuka, MSc; ${ }^{2}$ Gisele Peirano, $\mathrm{PhD} ;^{3}$ Corrinne Pidhorney, $\mathrm{RN} ;{ }^{2}$ Donna Ledgerwood, $\mathrm{MSc}^{2}$ Nancy Alfieri, BSc; ${ }^{2}$ Karen Hope, $\mathrm{MSc}^{2}$ Dan Gregson, MD; ${ }^{3,4,5,7}$ Johann Pitout, MD; $;^{3,4,6,7}$ Thomas Louie, $\mathrm{MD}^{2,5,6}$ John Conly, $\mathbf{M D}^{2,3,4,5,6,7}$}

Affiliations: 1. Department of Pediatrics, Alberta Children's Hospital, Alberta Health Services, Calgary, Alberta, Canada; 2. Infection Prevention and Control, Alberta Health Services-Calgary Zone (Alberta Children's Hospital, Foothills Medical Centre, Peter Lougheed Centre, Rockyview General Hospital), Calgary, Alberta, Canada; 3. Calgary Lab Services, Calgary, Alberta, Canada; 4. Department of Laboratory Medicine and Pathology, University of Calgary, Calgary, Alberta, Canada; 5. Department of Medicine, University of Calgary, Calgary, Alberta, Canada; 6. Department of Microbiology, Immunology, and Infectious Diseases, University of Calgary, Calgary, Alberta, Canada; 7. Snyder Institute for Chronic Diseases, University of Calgary, Calgary, Alberta, Canada.

Address correspondence to John Conly, MD, AGW5, Foothills Medical Centre, 1403-29th Street NW, Calgary, Alberta T2N 2T9, Canada (jconly@ucalgary.ca).

Infect Control Hosp Epidemiol 2014;35(4):450-451

(C) 2014 by The Society for Healthcare Epidemiology of America. All rights reserved. 0899-823X/2014/3504-0024\$15.00. DOI: $10.1086 / 675616$

\section{REFERENCES}

1. Kochar S, Sheard T, Sharma R, et al. Success of an infection control program to reduce the spread of carbapenem-resistant Klebsiella pneumoniae. Infect Control Hosp Epidemiol 2009;30: 447-452.

2. Schwaber MJ, Lev B, Israeli A, et al. Containment of a countrywide outbreak of carbapenem-resistant Klebsiella pneumoniae in Israeli hospitals via a nationally implemented intervention. Clin Infect Dis 2011;52:848-855.

3. Won SY, Munoz-Price LS, Lolans K, Hota B, Weinstein RA,
Hayden MK. Emergence and spread of Klebsiella pneumoniae carbapenemase-producing Enterobacteriaceae. Clin Infect Dis 2011:53:532-540.

4. Patel G, Huprikar S, Factor SH, Jenkins SG, Calfee DP. Outcomes of carbapenem-resistant Klebsiella pneumoniae infection and the impact of antimicrobial and adjunctive therapies. Infect Control Hosp Epidemiol 2008;29:1099-1106.

5. Jacob JT, Klein E, Laxminarayan R, et al. Vital signs: carbapenem-resistant Enterobacteriaceae. MMWR Morb Mortal Wkly Rep 62(09);165-170.

6. Brink A, Coetzee J, Clay C, et al. The spread of carbapenemresistant Enterobacteriaceae in South Africa: risk factors for acquisition and prevention. S Afr Med J 2102;102(7):599-601.

7. Calgary Economic Development. Demographics. http://www .calgaryeconomicdevelopment.com/research/ demographicspopulation/overview. Accessed August 8, 2013.

8. Government of Alberta. Tourism in Calgary area and tourism destination 2010: a summary of 2010 visitor numbers and characteristics. http://tpr.alberta.ca/tourism/statistics/docs /PersonvisitsCalgaryandArea2010.pdf. February 2012. Accessed October 8, 2013.

9. Conterno LO, Shymanski J, Ramotar K, Toye B, Zvonar R, Roth $V$. Impact and cost of infection control measures to reduce nosocomial transmission of extended spectrum B-lactamaseproducing organisms in a non-outbreak setting. J Hosp Inf 2007; $65: 354-360$.

10. Bank of Canada. Inflation calculator. http://www .bankofcanada.ca/rates/related/inflation-calculator/. Accessed April 28, 2013.

\section{Establishing Surveillance for Carbapenem- Resistant Enterobacteriaceae in Minnesota, 2012}

To the Editor-Carbapenem-resistant Enterobacteriaceae (CRE) have emerged as a public health threat; Enterobacteriaceae harboring carbapenemase-encoded genes often carry resistance to multiple antibiotic classes, rendering the bacteria resistant to almost all available antibiotics. Invasive CRE infections are associated with a high mortality rate $38 \%-$ $48 \%),{ }^{1-3}$ and intra- and interfacility spread in a variety of healthcare settings has occurred. ${ }^{4,5}$ A standardized approach to CRE surveillance and a clearer description of the evolving epidemiology are needed to understand the health burden and evaluate the impact of control measures.

In 2009, the Minnesota Department of Health (MDH) Public Health Laboratory (PHL) first confirmed Klebsiella pneumoniae carbapenemase in a clinical Enterobacteriaceae isolate. The MDH immediately initiated statewide voluntary reporting of CRE with isolate submission to the PHL (clinical cultures from all sources and Enterobacteriaceae species). In collaboration with the Centers for Disease Control and Prevention (CDC) Emerging Infections Program (EIP) Multisite resistant Gram-negative Surveillance Initiative (MuGSI), 